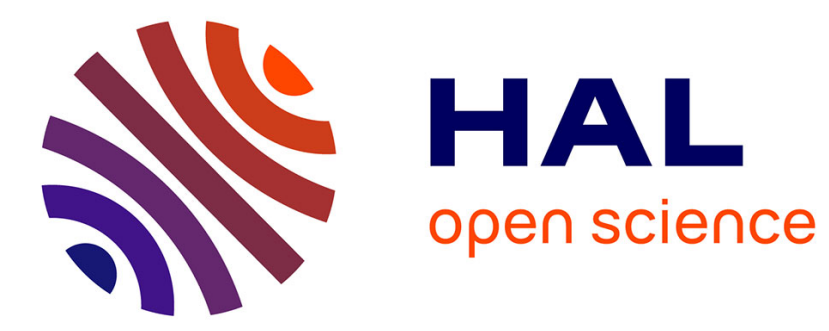

\title{
Lyapunov analysis of nonlinear systems with rational vector field and Jacobian
}

\author{
Ross Drummond, Giorgio Valmorbida, Stephen Duncan
}

\section{To cite this version:}

Ross Drummond, Giorgio Valmorbida, Stephen Duncan. Lyapunov analysis of nonlinear systems with rational vector field and Jacobian. 2016 UKACC 11th International Conference on Control (CONTROL), Aug 2016, Belfast, United Kingdom. 10.1109/CONTROL.2016.7737642 • hal-01710295

\section{HAL Id: hal-01710295 \\ https://hal.science/hal-01710295}

Submitted on 13 Apr 2020

HAL is a multi-disciplinary open access archive for the deposit and dissemination of scientific research documents, whether they are published or not. The documents may come from teaching and research institutions in France or abroad, or from public or private research centers.
L'archive ouverte pluridisciplinaire HAL, est destinée au dépôt et à la diffusion de documents scientifiques de niveau recherche, publiés ou non, émanant des établissements d'enseignement et de recherche français ou étrangers, des laboratoires publics ou privés. 


\section{Lyapunov Analysis of Nonlinear Systems With Rational Vector Field and Jacobian}

\author{
Ross Drummond \\ Dept. of Engineering Science \\ Universiy of Oxford \\ Oxford, UK, OX1 3PJ \\ ross.drummond@eng.ox.ac.uk
}

\author{
Giorgio Valmorbida \\ Laboratoire des Signaux \& Systèmes \\ CentraleSupélec, CNRS \\ Univ. Paris-Sud, Université Paris-Saclay \\ 3 Rue Joliot-Curie, Gif-sur-Yvette, 91192, France, \\ giorgio.valmorbida@12s.centralesupelec.fr
}

\author{
Stephen R. Duncan \\ Dept. of Engineering Science \\ Universiy of Oxford \\ Oxford, UK, OX1 3PJ \\ stephen.duncan@eng.ox.ac.uk
}

\begin{abstract}
This paper studies Lur'e type nonlinear systems where both the vector field and its Jacobian are rational with respect to the states and a sector bounded nonlinearities. Conditions to assess stability and compute induced $\mathscr{L}_{2}$-gain bounds for such systems are cast in terms of rational inequalities. A numerical solution for these inequalities is formulated as a convex optimisation problem given by a sum-of-squares program. Examples are given for nonlinear systems with the arctangent and rational nonlinearities. The proposed method is compared to the Popov and Circle criteria in a numerical example and is shown to outperform both of these classical results.
\end{abstract}

\section{INTRODUCTION}

Absolute stability theory is used to analyse particular forms of nonlinear systems called Lur'e systems. These systems can be expressed as the feedback interconnection of a linear system and a sector bounded nonlinearity [1]. The stability properties of this system can be studied via passivity properties of each term in the interconnection. If the interconnection is stable, a Lyapunov function can be constructed using the storage functions of the elements in the interconnection. The two main approaches to study absolute stability are the Lyapunov analysis approach and the frequency domain multiplier approach. A relation between multipliers and the Lyapunov function was established by the celebrated KYP Lemma [1] which led to the Popov and Circle criterions. Relatively recent developments in this field have been concerned with expressing these frequency domain conditions as linear matrix inequalities (LMIs) such that they can be checked by solving a convex optimisation problem [2].

The class of system that can be described as a Lur'e system is fairly large, with the only assumption on the nonlinearity being that it is sector bounded and decentralised. Another class of nonlinear system for which Lyapunov analysis has been succesfully applied, is the class of systems defined by rational vector fields studied with polynomial or rational Lyapunov functions (LF) [3]. Developments on convex optimisation have promoted the study of this class thanks to the use of Sumof-Squares programming (SOSP) [4] and SOSTOOLS [5]. Stability conditions for such systems can be checked (with some degree of conservatism [6]) using polynomial Lyapunov functions by the sum-of-squares (SOS) decomposition. Another common class of systems for which improved results exist are saturating systems. Examples of such results include the local analysis [7], [8], taking advantage of the boundedness of the nonlinearity [9] and using piece-wise polynomial LFs [10].

In this paper, Lur'e nonlinear systems where both the vector field and its Jacobian are rational functions of both the states and the nonlinearity are considered. This work was motivated by the practical application of computing lower bounds for the resistance of an electrochemical model of a supercapacitor [11]. Reducing the conservatism in these resistance bounds will enable designers to make improved predictions about the energy dissipation properties of the supercapacitor and allow improved supercapacitors to be designed using fewer experiments. The nonlinearity of this supercapacitor model is a logarithm which (locally) satisfies the requirements of the class of system considered.A reduced model of the supercapacitor is a Lur'e system and so can be analysed locally using the tools of absolute stability theory. In an extension to this paper, the results will be developed for a local analysis such that the supercapacitor model can be studied. The proposed method is a development for reducing the conservatism of the perturbation analysis by including information on the derivative of the nonlinearity directly into the conditions for the construction of a Lyapunov function (LF).

A relevant feature of the results presented in the paper is the fact that we use sum-of-squares programming to study non-polynomial systems. Generalised quadratic forms can also be used to study trigonometric systems [12] and polynomial approximations to the nonlinear functions were presented in [13], [14]. The approach presented in [15] proposes to bound the nonlinearities in a Lur'e system by polynomials, not by linear functions. This paper develops the authors' previous work on the analysis of Lur'e systems [16] by considering LFs which are polynomial, not just quadratic, with respect to the state and the nonlinearity.

The paper is structured as follows. Section II details the system with rational vector field and Jacobian with regards to the states and the nonlinearities. Section III gives inequality conditions for verifying global stability and for obtaining minimal upper bounds for the induced $\mathscr{L}_{2}$ gain of this system. Section IV describes the numerical implementation of these 
inequality conditions using SOS programming and presents a numerical example to evaluate the performance of the proposed method against the classical Circle and Popov methods. Finally, Section V presents some concluding remarks.

Notation A column vector of size $n$ containing ones is denoted $\mathbf{1}_{n} . y_{i}$ will denote the $i^{\text {th }}$ element of vector $y$ and $C_{i}$ will denote the $i^{t h}$ row of matrix $C$. Denote by $\mathscr{R}^{n}[x, \phi]$ the ring of polynomials of real coefficients in variables $x$ and $\phi$ on the real line and $\Sigma^{n}[x, \phi]$ by the ring of positive sum-ofsquares polynomials of dimension $n$. The set of non-negative real numbers is denoted $\mathbb{R}_{\geq 0}$. The set of symmetric matrix with real entries is denoted $\mathbb{S}$, and its subset, of diagonal matrices is denoted $\mathbb{D}$. The sets $\mathbb{S}_{\geq 0}, \mathbb{D}_{\geq 0}$ respectively denote the sets of positive semi-definite symmetric and diagonal matrices. We use $\|z\|=\int_{0}^{\infty} \sqrt{z^{T}(\theta) z(\theta)} \mathrm{d} \theta$.

\section{Problem Statement}

Consider the dynamical system

$$
\begin{aligned}
\dot{x} & =f(x, \phi(y), w) \\
y & =C x \\
z & =C_{z} x
\end{aligned}
$$

with state $x \in \mathbb{R}^{n}, w \in \mathbb{R}^{n_{w}}$ is a disturbance term and $z \in \mathbb{R}^{n_{z}}$ is the output, $\phi: \mathbb{R}^{m} \rightarrow \mathbb{R}^{m}$, and

$$
f(x, \phi(y), w)=\left(D_{1}(x, \phi(y))\right)^{-1} N_{1}(x, \phi(y))+B_{w} w
$$

where $N_{1}(x, \phi) \in \mathscr{R}^{n}[x, \phi], D_{1}(x, \phi) \in \mathscr{R}[x, \phi]$.

The class of nonlinearities considered will be restricted to those which are decentralised

$$
\phi(y)=\left[\begin{array}{lll}
\phi_{1}\left(y_{1}\right) & \phi_{2}\left(y_{2}\right) & \ldots \phi_{m}\left(y_{m}\right)
\end{array}\right]^{T},
$$

sector bounded

$$
\frac{\phi_{i}\left(y_{i}\right)}{y_{i}} \in\left[0, \overline{\delta_{i}}\right] \quad \forall y \in \mathbb{R}^{m}
$$

and satisfy

$$
\frac{\mathrm{d} \phi\left(y_{i}\right)}{\mathrm{d} y_{i}}=\left(D_{2 i}\left(\phi\left(y_{i}\right), y_{i}\right)^{-1} N_{2 i}\left(\phi\left(y_{i}\right), y_{i}\right) .\right.
$$

where $N_{2}\left(\phi_{i}, y_{i}\right) \in \mathscr{R}^{m}\left[\phi_{i}, y_{i}\right], D_{2}\left(\phi_{i}, y_{i}\right) \in \mathscr{R}\left[\phi_{i}, y_{i}\right]$.

If (4) holds, then

$$
s_{1}(y, \phi(y), T(x, \phi(y))):=(\phi(y)) T(x, \phi(y))(\bar{\Delta} y-\phi(y)) \geq 0
$$

where $T: \mathbb{R}^{n} \times \mathbb{R}^{m} \rightarrow \mathbb{D}_{\geq 0}^{m}, \bar{\Delta}=\operatorname{diag}\left(\overline{\boldsymbol{\delta}}_{1}, \ldots, \overline{\boldsymbol{\delta}}_{m}\right)$.

We propose solutions to the following problems:

Problem Statement 1 (Stability): Provide conditions to asses the global stability of the origin of system (1).

Problem Statement 2 ( $\mathscr{L}_{2}$ gain): Obtain upper bounds for the induced $\mathscr{L}_{2}$ gain from $w$ to $z$ of (1).

\section{MAIN RESULT}

In this section, conditions are outlined for solving Problems 1 and 2. The method is based upon the construction of a Lyapunov function which takes the form

$$
V(x)=V_{0}(x, \phi(y(x)))+\sum_{i=1}^{m} \lambda_{i} \int_{0}^{y_{i}(x)} \phi(\sigma) d \sigma .
$$

Such a function generalizes the Lyapunov function studied in [16]. Conditions for $V(x)$ to be positive definite are set by the following lemma

Lemma 3.1: Consider $V(x)$ as in (7). If there exists $\tilde{\lambda}_{i} \in \mathbb{R}_{\geq 0}$ such that $V(0,0)=0$

$$
\begin{aligned}
& \tilde{\lambda}_{i} \geq \lambda_{i} i=1, \ldots, m \\
& V_{0}(x, \phi(y(x)))-\sum_{i=1}^{m} \frac{1}{2} \tilde{\lambda}_{i} \bar{\delta}_{i} y_{i}^{2}(x)>0 \quad \forall x \in \mathbb{R}^{n}
\end{aligned}
$$

then $V(x)>0, \forall x \in \mathbb{R}^{n}$.

The proof follows the same steps as the proof of [16, Lemma 4], which is concerned with $V_{0}$ being quadratic on $x$ and $\phi$.

Remark 3.1: Note that positivity of the individual terms that construct $V$ in (7) is not required.

The following theorem provides solutions to Problems 1 and 2 .

Theorem 3.2: Suppose there exists functions $V_{0}, T: \mathbb{R}^{n} \times$ $\mathbb{R}^{m} \rightarrow \mathbb{D}_{\geq 0}$, and scalars $\tilde{\lambda}_{i} \in \mathbb{R}_{\geq 0}, \lambda_{i} \in \mathbb{R}, \gamma \in \mathbb{R}_{\geq 0}$, if and

$$
\begin{aligned}
& V_{0}(x, \phi(y(x)))-\sum_{j=1}^{m} \frac{1}{2} \tilde{\lambda}_{j} \bar{\delta}_{j} y_{j}^{2}(x)-s_{1}(x, \phi(y), T(x, \phi(y)))>0 \\
& -\left\langle\left[\begin{array}{c}
\nabla_{x} V \\
\nabla_{\phi} V
\end{array}\right],\left[\begin{array}{c}
\dot{x} \\
\dot{\phi}
\end{array}\right]\right\rangle-s_{1}(x, \phi(y), T(x, \phi(y)))-\Omega(z, w)>0
\end{aligned}
$$

hold with

a) $\Omega=0$ and $w=0$;

b) $\Omega(z, w)=-\eta w^{T} w+z^{T} z$

then

a) The origin of (1) is globally asymptotically stable.

b) The induced $\mathscr{L}_{2}$ gain from $w$ to $z$ is upper-bounded by $\sqrt{\eta}$, that is, $\frac{\|z\|}{\|w\|} \leq \sqrt{\eta}$ for all solutions of (1) satisfying $x(0)=0$.

The proof is analogous to that of Theorem 4.1 of [16].

\section{NUMERICAL FORMULATION}

In this section we present a numerical procedure, based on convex optimisation to obtain certificates for the global stability of (1), that takes advantage of the rational structure of (2) and (5). For comparison purposes, we recall the Lyapunov functions commonly used to study Lur'e type systems, that is the quadratic Lyapunov function associated to the Circle criterion

$$
V_{Q}(x)=x^{T} P x
$$


and the Lyapunov function including an integral term, associated to the Popov criterion

$$
V_{Q L}(x)=x^{T} P x+\sum_{i=1}^{m} \lambda_{i} \int^{y} \phi(\sigma) d \sigma .
$$

To enforce positivity of the above function, we require $P>0$ and typically that the scalars of the integral term are set to be non-negative $\lambda_{i} \geq 0$ for $i=1, \ldots, m$.

\section{A. SOS formulation}

We now present a SOSP that solves the inequalities conditions of Theorem 3.2. We briefly recall some concepts related to SOS optimisation, along the lines of [17]:

Definition 1: A multivariate polynomial $p$ is said to be SOS polynomial if there exists a finite set of polynomials $h_{i} \in \mathscr{R}[x]$ satisfying

$$
p(x)=\sum_{i=1}^{M} h_{i}^{2}(x) .
$$

It was shown in [18] that the search for an SOS decomposition, i.e. the search for polynomials $h_{i}$ satisfying (12), could be set up as a convex Semi-Definite Program (SDP.)

Proposition 1: A polynomial $p(x) \in \mathscr{R}[x]$ of degree $2 d$ is an SOS polynomial if and only if there exists $Q \in \mathbb{S}_{\geq 0}$ and a vector of monomials $Z(x) \in \mathscr{R}^{M}[x]$ such that

$$
p(x)=Z(x)^{T} Q Z(x)
$$

then $p(x)$ is SOS.

If the coefficients of polynomial $p$ have an affine dependence on a set of decision variables $\{\eta, R\}$, then the following problem is a convex semi-definite problem

$$
\text { minimize } \quad \eta \quad \text { subject to } p(x, \eta, R) \in \Sigma[x] \text {. }
$$

As well as stability analysis, SOSP have been used to study reachability sets, controller synthesis [19], and $\mathscr{L}_{2}$ gains amongst other properties for systems described by polynomial vector fields in the states [17]. For rational inequalities, a polynomial inequality can be obtained by multiplying the rational expression by its denominator. The following proposition gives a SOSP relaxation of polynomial inequalities obtained from the rational inequalities (9) thus providing a sufficient condition to verify the inequalities of Theorem 3.2.

Proposition 2: If there exists $V_{0} \in \mathscr{R}[x, \phi], V(0,0)=0$, $T_{1}, \quad T_{2} \in \Sigma_{m}^{\text {diag }}[x, \phi], \quad \lambda_{i}, \tilde{\lambda}_{i} \in \mathbb{R}_{\geq 0}$, satisfying $\tilde{\lambda}_{i} \geq \lambda_{i} i=$ $1, \ldots, m, \eta \in \mathbb{R}_{\geq 0}$

$$
\begin{aligned}
& V_{0}-\sum_{i=1}^{m} \frac{1}{2} \tilde{\lambda}_{i} \bar{\delta}_{i} y_{i}^{2}(x)-s_{1}\left(y(x), \phi, T_{1}\right)-\varepsilon x^{T} x \in \Sigma[x, \phi] \\
& -\left\langle\left[\begin{array}{c}
\nabla_{x} V \\
\nabla_{\phi} V
\end{array}\right],\left[\begin{array}{c}
D_{2}(\phi, y)\left(N_{1}(\phi(y), y(x))+D_{1}(\phi, y(x)) B_{w} w\right) \\
D_{1}(\phi, y(x)) N_{2}(\phi, y(x)) \dot{y}(x)
\end{array}\right]\right\rangle \\
& -s_{1}\left(y(x), \phi, T_{2}\right)+D_{1}(\phi, y(x)) D_{2}(\phi, y(x))\left(\eta w^{T} w-z^{T}(x) z(x)\right) \\
& -\varepsilon x^{T} x \in \Sigma[x, \phi, w]
\end{aligned}
$$

with $D_{2}(\phi, y(x))=\prod_{i=1}^{m} D_{2 i}\left(\phi_{i}, y_{i}(x)\right)$ and $N_{2}(\phi, y(x))=$ $\operatorname{diag}\left(N_{21}\left(\phi_{1}, y_{1}(x), \ldots, N_{2 m}\left(\phi_{m}, y_{m}(x)\right)\right.\right.$ then the origin of (1) is

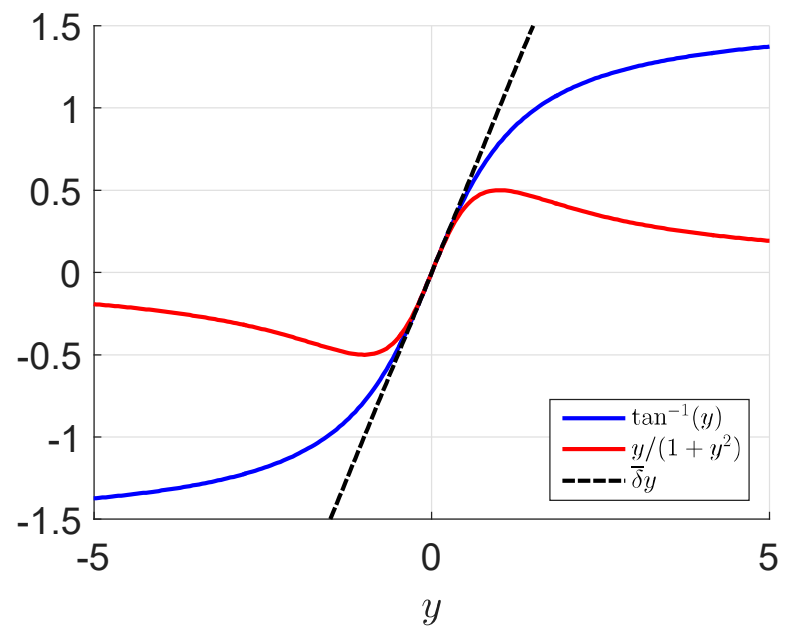

Figure 1. The shifted logarithm and arctangent functions plotted with local sector bounds.

globally asymptotically stable for $w \equiv 0$ and an upper-bound for the induced $\mathscr{L}_{2}$ gain of (1) is given by $\gamma=\sqrt{\eta}$ for all solutions of (1) satisfying $x(0)=0$.

To make the conditions of Proposition 2 verifiable using convex programming, the inequalities have been relaxed to be SOS constraints. The denominators of the rational terms that appear in $\dot{V}$ due to the rational vector field and Jacobian are cancelled by multiplying through its denominator, yielding (15b). The sign of the expression is preserved only in case $D_{1}(x, \phi) D_{2}(x, \phi)>0 \forall(x, \phi) \in \mathbb{R}^{n+m}$. We solve the following convex optimization to obtain a solution to Problem 2

$\min \eta$ subject to (15).

\section{B. Numerical Example}

Consider the following system

$$
\begin{aligned}
{\left[\begin{array}{l}
\dot{x}_{1} \\
\dot{x}_{2}
\end{array}\right] } & =\left[\begin{array}{cc}
0 & 1 \\
-2 & -1
\end{array}\right]\left[\begin{array}{l}
x_{1} \\
x_{2}
\end{array}\right]+\left[\begin{array}{c}
\frac{y}{1+y^{2}} \\
0
\end{array}\right]+\left[\begin{array}{l}
0 \\
1
\end{array}\right] \phi(y)+\left[\begin{array}{l}
0 \\
1
\end{array}\right] w \\
y & =\left[\begin{array}{ll}
-1 & 0
\end{array}\right]\left[\begin{array}{l}
x_{1} \\
x_{2}
\end{array}\right] \\
z & =\left[\begin{array}{ll}
1 & 0
\end{array}\right]\left[\begin{array}{l}
x_{1} \\
x_{2}
\end{array}\right] \\
\phi(y) & =\tan ^{-1}(y) .
\end{aligned}
$$

Both nonlinear terms of (20), corresponding to the arctangent $\tan ^{-1}$ and the rational term $y /\left(1+y^{2}\right)$, satisfy the properties of the sector bounded Lur'e system and of the polynomial system (1). These nonlinearities satisfy the $[0,1]$ sector condition of (4) and are plotted in Fig 1. The Jacobian of $\tan ^{-1}$ is a rational polynomial with respect to its argument

$$
\frac{\mathrm{d} \tan ^{-1}(y)}{\mathrm{d} y}=\frac{1}{1+y^{2}},
$$




\begin{tabular}{|c|c|}
\hline & $\gamma$ \\
\hline Linear & 1.000 \\
\hline$V_{Q}$ (sector) & 4.8160 \\
$V_{Q L}$ (sector) & 2.6069 \\
\hline$V_{Q}$ (multiply) & 1.6035 \\
$V_{Q L}$ (multiply) & 1.3710 \\
\hline \multicolumn{2}{|c|}{$V$} \\
\hline \multicolumn{2}{|c|}{ Table I } \\
\hline
\end{tabular}

INDUCED $\mathscr{L}_{2}$ GAINS $\gamma$ FOR (20) USING BOTH CASES OF THE POPOV AND CirCle CRITERIONS, PROPOSITION 2 AND FOR THE LINEAR SYSTEM WITHOUT ANY NONLINEARITIES.

which gives $D_{2}(\phi(y), y)=1+y^{2}$ in (5). The rational term is expressed as $N_{1}(x, \phi(y))=y$ and $D_{1}(x, \phi(y))=1+y^{2}$ with Jacobian

$$
\frac{\mathrm{d}\left(y /\left(1+y^{2}\right)\right)}{\mathrm{d} y}=\frac{\left(1-y^{2}\right)}{\left(1+y^{2}\right)^{2}} .
$$

which gives $D_{2}(\phi, y)=\left(1+y^{2}\right)^{2}$.

Upper bounds for $\gamma$ obtained with the Popov and Circle criteria as well as the proposed method are given in Tab. I. The linear case in this table considers (20) without either the arctangent or the rational term with dynamics

$$
\begin{aligned}
{\left[\begin{array}{c}
\dot{x}_{1} \\
\dot{x}_{2}
\end{array}\right] } & =\left[\begin{array}{cc}
0 & 1 \\
-2 & -1
\end{array}\right]\left[\begin{array}{l}
x_{1} \\
x_{2}
\end{array}\right]+\left[\begin{array}{l}
0 \\
1
\end{array}\right] w \\
z & =\left[\begin{array}{ll}
1 & 0
\end{array}\right]\left[\begin{array}{l}
x_{1} \\
x_{2}
\end{array}\right] .
\end{aligned}
$$

In the table we have considered two approaches while using the functions $V_{Q}$ and $V_{Q L}$. For the case (sector), both the rational term and the arctangent are described solely by sector conditions while for the case (multiply), sector information is used to characterise the arctangent but not the rational term. In this case, the terms appearing in the denominator of $\dot{V}_{Q L}$ and $\dot{V}_{Q}$ are canceled out by multiplying through by $D_{1}(x, \phi(y))$. Comparing the values of $\gamma$ given in Tab. I, the proposed method outperforms both implementations of the Popov and Circle criteria. Note that for this particular system the bound given by Proposition 2 is the same as that of the linear system, which implies that the worst case performance with the nonlinearities is the same as the open loop system. The characterisation of the rational terms using sector information was more conservative than by multiplying through by the denominator $D_{1}(x, \phi(y))$. This result is due to the conservatism of the sector conditions, whose linear approximations of the nonlinearities are inaccurate far away form the origin.

\section{CONCLUSION}

This papers has considered nonlinear systems where both the vector field and its Jacobian are rational functions of the states and a nonlinearity. Numerical solutions to the proposed stability and performance assessment conditions are obtained using sum-of-squares programming. A numerical example has illustrated the proposed result for a system with a rational polynomial and the arctangent function. The proposed method was able to compute upper-bounds for the induced $\mathscr{L}_{2}$ gain of such a system, improving upon the results obtained with the Popov and Circle criteria. This improved performance is due to the fact that the proposed method includes information about the slope of the nonlinearity directly into the construction of a Lyapunov function by explicitly writing its Jacobian. This improves upon the characterisation of the nonlinearity only by sector information.

\section{ACKNOWLEDGMENT}

This work was supported by the UK Engineering and Physical Sciences Research Council

\section{REFERENCES}

[1] HK Khalil. Nonlinear Systems. Prentice hall New Jersey, 3 edition, 2002.

[2] S Boyd and L Vandenberghe. Convex Optimization. Cambridge university press, 2004.

[3] G Valmorbida and J Anderson. Region of attraction analysis via invariant sets. In Proc of the $32^{\text {nd }}$ American Control Conference, 2014, pages 3591-3596. IEEE, 2014.

[4] A Papachristodoulou and S Prajna. On the construction of Lyapunov functions using the sum of squares decomposition. In Proc.of the $41^{s t}$ IEEE Conference on Decision and Control, pages 3482-3487. IEEE, 2002.

[5] A Papachristodoulou, J Anderson, G Valmorbida, S Prajna, P Seiler, and P Parrilo. SOSTOOLS version 3.00 sum of squares optimization toolbox for MATLAB. arXiv preprint arXiv:1310.4716, 2013

[6] AA Ahmadi, M Krstic, and PA Parrilo. A globally asymptotically stable polynomial vector field with no polynomial Lyapunov function. In Proc. of 50 ${ }^{\text {th }}$ IEEE Conference on Decision and Control, pages 7579-7580. IEEE, 2011

[7] H Hindi and S Boyd. Analysis of linear systems with saturation using convex optimization. In Proc. of the $37^{\text {th }}$ IEEE Conference on Decision and Control, pages 903-908. IEEE, 1998.

[8] C Pittet, S Tarbouriech, and C Burgat. Stability regions for linear systems with saturating controls via Circle and Popov criteria. In Proc of the $36^{\text {th }}$ IEEE Conference on Decision and Control, pages 4518-4523. IEEE 1997.

[9] M Saeki, N Wada, and S Satoh. Stability analysis of feedback systems with dead-zone nonlinearities by circle and Popov criteria. Automatica, 66:96-100, 2016

[10] A Garulli, A Masi, G Valmorbida, and L Zaccarian. Global stability and finite $\mathscr{L}_{2}$ gain of saturated uncertain systems via piecewise polynomial Lyapunov functions. IEEE Transactions on Automatic Control, 1(58):242-246, 2013.

[11] R Drummond, DA Howey, and SR Duncan. Low-order mathematical modelling of electric double layer supercapacitors using spectral methods. Journal of Power Sources, 277:317-328, 2015.

[12] A Papachristodoulou and S Prajna. Analysis of non-polynomial systems using the sum of squares decomposition. In Positive Polynomials in Control, pages 23-43. Springer, 2005.

[13] S Mastellone, PF Hokayem, CT Abdallah, and P Dorato. Nonlinear stability analysis for non-polynomial systems. In Proc. of $22^{\text {nd }}$ IEEE American Control Conference, pages 1725-1730. IEEE, 2004.

[14] G Chesi. Estimating the domain of attraction for non-polynomial systems via LMI optimizations. Automatica, 45(6):1536-1541, 2009.

[15] EJ Hancock and A Papachristodoulou. Generalised absolute stability and sum of squares. Automatica, 49(4):960-967, 2013.

[16] G Valmorbida, R Drummond, and SR Duncan. Positivity conditions of Lyapunov functions for systems with slope restricted nonlinearities. To appear in Proc. of the $34^{\text {th }}$ IEEE American Control Conference. IEEE, 2016.

[17] A Papachristodoulou and S Prajna. A tutorial on sum of squares techniques for systems analysis. In Proc.of the $22^{\text {nd }}$ IEEE American Control Conference, pages 2686-2700. IEEE, 2005.

[18] PA Parrilo. Structured semidefinite programs and semialgebraic geometry methods in robustness and optimization. $\mathrm{PhD}$ thesis, 2000.

[19] G Valmorbida, S Tarbouriech, and G Garcia. Design of polynomial control laws for polynomial systems subject to actuator saturation. IEEE Transactions on Automatic Control, 58(7):1758-1770, 2013. 